\title{
Noether symmetries and isometries of the minimal surface Lagrangian under constant volume in a Riemannian space
}

\author{
MICHAEL TSAMPARLIS \\ Faculty of Physics, Department of Astrophysics - Astronomy - Mechanics, University of Athens, \\ Panepistemiopolis, Athens 157 83, Greece \\ mtsampa@phys.uoa.gr \\ ANDRONIKOS PALIATHANASIS \\ Dipartimento di Fisica, Universita' di Napoli, "Federico II", Compl. Univ. di Monte S. Angelo, \\ Via Cinthia, I-80126, Napoli, Italy \\ INFN Sez. di Napoli, Compl. Univ. di Monte S. Angelo, Via Cinthia, I-80126, Napoli, Italy \\ Faculty of Physics, Department of Astrophysics - Astronomy - Mechanics, University of Athens, \\ Panepistemiopolis, Athens 157 83, Greece \\ paliathanasis@na.infn.it \\ ASGHAR QADIR \\ School of Natural Sciences, National University of Sciences and Technology, Islamabad, \\ Pakistan \\ asgharqadir46@gmail.com
}

\begin{abstract}
We prove a theorem concerning the Noether symmetries for the area minimizing Lagrangian under the constraint of a constant volume in an $n$-dimensional Riemannian space. We illustrate the application of the theorem by a number of examples.
\end{abstract}

Keywords: Noether symmetries; Minimal Surfaces

\section{Introduction}

The principle of equivalence, although expressed explicitly in General Relativity, is usually implicitly assumed in dynamical system theory. According to this principle free motion of a dynamical system occurs along the geodesics of the background space where motion takes place. In other words the geometry of the background space determines uniquely the kinematics of the dynamical system. This is why the geodesic equations of a space are so important in the study of the evolution of dynamical systems. The second factor which makes these equations important is that when a dynamical system is "moving" under the action of a "force" then the only change in the equations of "motion" is that the geodesic equations from homogeneous differential equations become inhomogeneous, the inhomogeneity factor being the force in the right hand side of the equations. In short the geodesic equations of a space are important to study the evolution of dynamical systems. 
The above considerations led to the study of Lie symmetries of second order ordinary differential equations (ODEs). In a number of papers [1|2]3 it has been shown that the Lie symmetries of the geodesic equations are elements of the projective algebra of the space. Furthermore, because the geodesic equations follow from the geodesic Lagrangian, people have studied the Noether symmetries of this Lagrangian and showed that the Noether symmetries are elements of the homothetic algebra of the space [4. The knowledge of Noether symmetries is important because it provides the Noether currents which can be used for a double reduction of the equations of motion and, if there is a sufficient number of them, to the solution of the ODE by means of quadratures.

These studies have been generalized to Lie and the Noether symmetries of certain classes of partial differential equations (PDEs) [5/6/78] and it has been shown that their generators are from the conformal group of the background space.

The Noether point symmetries are symmetries of the action integral. Therefore in principle they concern all problems involving an "action" integral even if these problems do not concern the equations of motion. One such problem is the determination of the minimal surface area under constant volume in a given Riemannian space. In this case the "action" integral involves the minimization of a surface and not of an arc length, as is the case with the equations of motion [9|10[1]. In [1] the authors have found a relation between the Noether symmetries of the minimal Lagrangian with the isometries of the underlying space for some specific spaces. The purpose of this work it to give a geometric proof of this result and generalize the problem in two directions: (a) in a general Riemannian space (having the appropriate topology in order closed surfaces with a finite volume to exist); and (b) in a dynamical way, that goes beyond geometry, in General Relativity and dynamical systems theory. The plan of the paper is as follows.

In section 2, we present the necessary definitions and notation to be used. In section 3 we determine the Noether point symmetries of the minimal surface action under constant volume and show that the generators of Noether symmetries are elements of the Killing algebra of the space. Due to the two dimensionality of the variation we have conservation currents instead of simple first integrals together with two extra conditions. In section 4 we apply our results to a general Euclidian space, spaces of constant curvature and to Schwarzschild spacetime. We also show how these results can be used to reduce the minimal surface equation form a PDE to an ODE in the FRW spacetime with dust. Finally in section 5 we draw our conclusions.

\section{Preliminaries}

A partial differential equation (PDE) is a function $H=H\left(x^{i}, u^{A}, u_{, i}^{A}, u_{, i j}^{A}\right)$ in the jet space $\bar{B}_{\bar{M}}$, where $x^{i}$ are the independent variables and $u^{A}$ are the dependent 
variables. The infinitesimal point transformation

$$
\begin{aligned}
\bar{x}^{i} & =x^{i}+\varepsilon \xi^{i}\left(x^{k}, u^{B}\right), \\
\bar{u}^{A} & =\bar{u}^{A}+\varepsilon \eta^{A}\left(x^{k}, u^{B}\right),
\end{aligned}
$$

has the infinitesimal symmetry generator

$$
\mathbf{X}=\xi^{i}\left(x^{k}, u^{B}\right) \partial_{x^{i}}+\eta^{A}\left(x^{k}, u^{B}\right) \partial_{u^{A}} .
$$

$\mathbf{X}$ is called a Lie point symmetry of the PDE $H$ if there exists a function $\lambda$ such that the following condition holds [12]13]

$$
\mathbf{X}^{[n]}(H)=\lambda H \quad, \quad \bmod H=0,
$$

where

$$
\mathbf{X}^{[n]}=\mathbf{X}+\eta_{i}^{A} \partial_{\dot{x}^{i}}+\eta_{i j}^{A} \partial_{u_{i j \ldots i_{n}}^{A}}+\ldots+\eta_{i_{1} i_{2} \ldots i_{n}}^{A} \partial_{u_{i_{1} i_{2} \ldots i_{n}}^{A}}
$$

is the $n^{\text {th }}$ prolongation vector and

$$
\eta_{i}^{A}=\eta_{, i}^{A}+u_{, i}^{B} \eta_{, B}^{A}-\xi_{, i}^{j} u_{, j}^{A}-u_{, i}^{A} u_{, j}^{B} \xi_{, B}^{j},
$$

with

$$
\begin{aligned}
\eta_{i j}^{A} & =\eta_{, i j}^{A}+2 \eta_{, B(i}^{A} u_{, j)}^{B}-\xi_{, i j}^{k} u_{, k}^{A}+\eta_{, B C}^{A} u_{, i}^{B} u_{, j}^{C}-2 \xi_{,(i|B|}^{k} u_{j)}^{B} u_{, k}^{A} \\
& -\xi_{, B C}^{k} u_{, i}^{B} u_{, j}^{C} u_{, k}^{A}+\eta_{, B}^{A} u_{, i j}^{B}-2 \xi_{,(j}^{k} u_{, i) k}^{A}-\xi_{, B}^{k}\left(u_{, k}^{A} u_{, i j}^{B}+2 u_{(, j}^{B} u_{, i) k}^{A}\right)
\end{aligned}
$$

For PDEs arising from a variational principle Noether's theorem [14] states

Theorem 1. The action of transformation (1) on the Lagrangian $L=$ $L\left(x^{k}, u^{A}, u_{k}^{A}\right)$ leaves $H\left(x^{i}, u^{A}, u_{, i}^{A}, u_{, i j}^{A}\right)$ invariant if there exists a vector field $A^{i}=$ $A^{i}\left(x^{i}, u^{A}\right)$ such that the following condition is satisfied,

$$
\mathbf{X}^{[1]} L+L D_{i} \xi^{i}=D_{i} A^{i} .
$$

The corresponding Noether flow is the divergence-free vector

$$
I^{i}=\xi^{k}\left(u_{k} \frac{\partial L}{\partial u_{i}}-L\right)-\eta \frac{\partial L}{\partial u_{i}}+A^{i} .
$$

where $D_{i}$ is total derivative

$$
D_{i}=\partial_{x^{i}}+u_{i}^{A} \partial_{u^{A}}+u_{i j}^{A} \partial_{u_{j}^{A}}+\ldots
$$

We now proceed to study the Noether point symmetries of the minimal surface Lagrangian 
M. Tsamparlis, A. Paliathanasis \& A. Qadir

\section{Noether symmetries of the minimal surface Lagrangian under constant volume}

Consider a Riemannian space $V^{n}$ with line element

$$
d s^{2}=d u^{2}+h_{i j}\left(u, x^{k}\right) d x^{i} d x^{j}
$$

where $i, j=1,2, \ldots, n-1$. This metric is not in general $1+(n-1)$ decomposable because $h_{i j}$ is a function of $u$ as well as $x^{k}$. However if there exist a coordinate system such that $h_{i j}=h_{i j}\left(x^{k}\right)$ then (9) admits the gradient $\mathrm{KV} \partial_{u}$ and it is $1+(n-1)$ decomposable.

The Lagrangian of the minimal surface enclosing a constant volume is [1]

$$
L=\sqrt{|h|+|h| h^{i j} u_{, i} u_{, j}}+\lambda \int \sqrt{|h|} d u .
$$

For this Lagrangian the Noether symmetry condition gives

$$
\begin{aligned}
0 & =|h|_{, k} \xi^{k}+|h|_{, k} \xi^{k} h^{i j} u_{, i} u_{, j}+|h| h_{, k}^{i j} \xi^{k} u_{, i} u_{, j}+|h|_{, u} \eta+|h|_{, u} \eta h^{i j} u_{, i} u_{, j}+|h| h_{, u}^{i j} \eta u_{, i} u_{, j} \\
& +2|h| h^{i k} u_{, k}\left(\eta_{, i}+u_{i} \eta_{u}-\xi_{, i}^{r} u_{r}\right)+2|h|\left(\xi_{, k}^{k}+\xi_{, u}^{k} u_{k}\right)+2|h| h^{i j} u_{, i} u_{, j} \xi_{, k}^{k} \\
& \lambda\left(\int \sqrt{|h|} d u\right)_{k} \xi^{k}+\lambda \sqrt{|h|} \eta+\lambda\left(\int \sqrt{|h|} d u\right)\left(\xi_{, k}^{k}+\xi_{, u}^{k} u_{k}\right)=A_{, k}^{k}+A_{, u}^{k} u_{k} .
\end{aligned}
$$

In (11) comparing the coefficients of powers of $u_{i}$ we find

$$
\begin{gathered}
\left(u_{i}\right)^{0}: \quad|h|_{, u} \eta+2|h|_{; ; k}^{k}=0 ; \\
\left(u_{i}\right): \quad h^{i k} \eta_{, i}+\xi_{, u}^{k}=0
\end{gathered}
$$

$\left(u_{i} u_{j}\right): \quad|h|\left(h_{, k}^{i j} \xi^{k}-2 \xi^{(i, k)}\right)+\left(2|h| h^{i j} \xi_{, k}^{k}+|h|_{, k} \xi^{k} h^{i j}+|h|_{, u} \eta h^{i j}\right)+|h| h_{, u}^{i j} \eta+2|h| h^{i k} \eta_{, u}=0$.

The third condition yields

$$
-2 \xi^{(i ; j)}+h_{, u}^{i j} \eta+2 h^{i k} \eta_{, u}=0
$$

where the covariant derivative is with respect to the metric $h_{i j}$ in the $n-1$ space $\left\{x^{i}\right\}$.

Similarly, comparing coefficients in (12) we get

$$
\begin{gathered}
\left(u_{i}\right)^{0}: \quad \lambda\left(\int \sqrt{|h|} d u\right)_{k} \xi^{k}+\lambda \sqrt{|h|} \eta+\lambda\left(\int \sqrt{|h|} d u\right) \xi_{, k}^{k}=A_{, k}^{k} ; \\
u_{i}: \quad \lambda\left(\int \sqrt{|h|} d u\right) \xi_{, u}^{k}=A_{, u}^{k} .
\end{gathered}
$$

To obtain $\mathbf{X}$ and $\mathbf{A}$ we need to solve the resulting Noether symmetry conditions for the above Lagrangian.

Contracting (16) with $h_{i j}$ and using (13) we find $\eta_{, u}=0$, i.e. $\eta$ is a function of $x^{i}$ only. Thus (16) reduces to

$$
2 \xi^{(i ; j)}=\eta h_{, u}^{i j}
$$


Now for the metric given above

$$
L_{\mathbf{X}} g_{a b}=\left[h_{i j, u} \eta+2 \xi_{(i ; j)}\right] \delta_{b}^{j} \delta_{a}^{i}+2\left[\xi_{, u}^{i} h_{j i}+\eta_{, j}\right] \delta_{b}^{j} \delta_{a}^{u}+2 \eta_{, u} \delta_{b}^{u} \delta_{a}^{u} .
$$

As $\eta$ is not a function of $u$ we have the condition

$$
L_{\mathbf{X}} g_{a b}=0 .
$$

In other words, the Noether vector for the Lagrangian is a Killing vector of the $n$ dimensional metric.

For the gauge vector the condition (18) reduces to

$$
A^{k}=\lambda \int\left[\left(\int \sqrt{|h|} d u\right) \xi_{, u}^{k}\right] d u+\Phi^{k}\left(x^{i}\right)
$$

where $\Phi^{k}\left(x^{k}\right)$ is an arbitrary function of its argument, which must, by (17) satisfy

$$
\Phi_{, k}^{k}=\lambda\left(\int \sqrt{|h|} d u \xi^{k}\right)_{, k}+\lambda \sqrt{|h|} \eta-\lambda\left[\int\left(\int \sqrt{|h|} d u\right) \xi_{, u}^{k} d u\right]_{, k} .
$$

Therefore concerning the Noether symmetries of the minimal surface Lagrangian we have the following theorem:

Theorem 2. The Noether point symmetries of the Lagrangian of the minimal surface enclosing a constant volume in a space are the Killing vector fields $\mathbf{X}=$ $\xi^{i}\left(x^{k}, u\right) \partial_{i}+\eta\left(x^{k}\right) \partial_{u}$ of the space, provided there exists a vector field $A^{i}\left(u, x^{k}\right)$ given by the expression

$$
A^{k}=\lambda \int\left(\int \sqrt{|h|} d u\right) \xi_{, u}^{k} d u+\Phi^{k}\left(x^{k}\right)
$$

where

$$
\Phi_{, k}^{k}=\lambda\left(\int \sqrt{|h|} d u \xi^{k}\right)_{, k}+\lambda \sqrt{|h|} \eta-\lambda\left[\int\left(\int \sqrt{|h|} d u\right) \xi_{, u}^{k} d u\right]_{, k} .
$$

In the case of the minimal surface Lagrangian without the constraint of constant volume, that is when $\lambda=0$, we have the following results:

Corollary 3. The minimal surface Lagrangian

$$
L=\sqrt{|h|+|h| h^{i j} u_{, i} u, j},
$$

admits as Noether point symmetries the vector field $\mathbf{X}=\xi^{i}\left(x^{k}, u\right) \partial_{i}+\eta\left(x^{k}\right) \partial_{u}$, which is the generic Killing vector of the space with metric (9). The corresponding Noether gauge field satisfies the conditions $A_{, k}^{k}=0$ and $A_{, u}^{k}=0$.

Corollary 4. If the minimal surface Lagrangian admits the $G_{N}$ Noether algebra, then $\max \left(\operatorname{dim} G_{N}\right)=\frac{1}{2} n(n+1)$ if and only if the space is a maximally symmetric space, i.e. has constant curvature. 


\section{Applications}

In this section we apply theorem 2 to determine the Noether point symmetries of the minimal surface Lagrangian (10) in some interesting cases.

\subsection{The Euclidian case}

For simplicity and in order to demonstrate the application of theorem 2 we consider a surface $r=r(\theta, \phi)$ in $3 \mathrm{~d}$ Euclidian space $E^{3}$, whose metric in spherical coordinates $r, \theta, \phi$ is:

$$
d s^{2}=d r^{2}+r^{2} d \theta^{2}+r^{2} \sin ^{2} \theta d \phi^{2} .
$$

Obviously the restriction to dimension 3 is of no importance. The metric $h_{i j}=$ $\operatorname{diag}\left(r^{2}, r^{2} \sin ^{2} \theta\right)$ yields $|h|=r^{4} \sin ^{2} \theta$. The Lagrangian of the minimal surface enclosing a constant volume is

$$
L=\sqrt{r^{4} \sin ^{2} \theta+r^{2} \sin ^{2} \theta r_{, \theta}^{2}+r^{2} r_{, \phi}^{2}}+\frac{\lambda}{3} r^{3} \sin \theta .
$$

The KVs of $E^{3}$ in spherical coordinates are

a. The subalgebra of rotations $S O(3)$ :

$$
\begin{aligned}
& K^{1}=\sin \phi \partial_{\theta}+\cot \theta \cos \phi \partial_{\phi}, \\
& K^{2}=\cos \phi \partial_{\theta}-\cot \theta \sin \phi \partial_{\phi}, \\
& K^{3}=\partial_{\phi} .
\end{aligned}
$$

b. The subalgebra of translations $T(3)$ :

$$
\begin{aligned}
& T^{1}=\sin \phi \sin \theta \partial_{r}+\frac{\cos \theta \sin \phi}{r} \partial_{\theta}+\frac{\cos \phi}{r \sin \theta} \partial_{\phi}, \\
& T^{2}=\cos \phi \sin \theta \partial_{r}+\frac{\cos \theta \cos \phi}{r} \partial_{\theta}-\frac{\sin \phi}{r \sin \theta} \partial_{\phi}, \\
& T^{3}=\cos \theta \partial_{r}-\frac{\sin \theta}{r} \partial_{\theta} .
\end{aligned}
$$

From (24) we compute the Noether gauge vectors $A^{k}$. We have

$$
\int \sqrt{|h|} d u=\int r^{2} \sin \theta d r=\frac{1}{3} r^{3} \sin \theta .
$$

therefore

$$
A^{k}=\frac{\lambda}{3} \sin \theta \int r^{3} \xi_{, r}^{k} d r+\Phi^{k}\left(x^{k}\right)
$$

where $\xi^{k}$ is the projection of any $\mathrm{KV}, X^{a}$, in the $\theta, \phi$ space where $\Phi^{k}(\theta, \phi)$ satisfies (25).

For the translation $T^{1}$ we have

$$
\eta=\sin \phi \sin \theta, \xi^{i}=\frac{\cos \theta \sin \phi}{r} \partial_{\theta}+\frac{\cos \phi}{r \sin \theta} \partial_{\phi} .
$$


hence

$$
A^{i}\left(T_{1}\right)=-\frac{\lambda}{6} r^{2}\left(\sin \theta \cos \theta \sin \phi \partial_{\theta}+\cos \phi \partial_{\phi}\right)+\Phi^{i}(\theta, \phi) .
$$

Similarly we compute

$$
\begin{aligned}
& A^{i}\left(T_{2}\right)=\frac{\lambda}{6} r^{2}\left(\sin \theta \cos \theta \cos \phi \partial_{\theta}-\sin \phi \partial_{\phi}\right)+\Phi^{i}(\theta, \phi) \\
& A^{i}\left(T_{3}\right)=-\frac{\lambda}{6} r^{2} \sin ^{2} \theta \partial_{\theta}+\Phi^{i}(\theta, \phi)
\end{aligned}
$$

The rotations give zero gauge fields. These results agree with those of [1].

\subsection{Spaces of constant curvature}

We consider next the surface $\theta=\theta(\phi, \psi)$ in $3 \mathrm{~d}$ Euclidian space $S^{3}$ in spherical coordinates $\theta, \phi, \psi$ with metric:

$$
d s^{2}=d \theta^{2}+\sin ^{2} \theta\left(d \phi^{2}+\sin ^{2} \phi d \psi^{2}\right) .
$$

Obviously again the restriction to $3 \mathrm{~d}$ is not important. In this case the metric $h_{i j}=\operatorname{diag}\left(\sin ^{2} \theta, \sin ^{2} \phi\right)$ and hence $|h|=\sin ^{4} \theta \sin ^{2} \phi$.

The Lagrangian of the minimal surface under constant volume is

$$
L=\sqrt{\sin ^{4} \theta \sin ^{2} \phi+\sin ^{2} \theta \sin ^{2} \phi \theta_{, \phi}^{2}+\sin ^{2} \theta \theta_{, \psi}^{2}}+\frac{\lambda}{2} \sin \phi\left(\theta-\frac{1}{2} \sin (2 \theta)\right) .
$$

The $S^{3}$ admits six non-gradient KVs,

$$
\begin{aligned}
& X_{1}=\sin \phi \sin \psi \partial_{\theta}+\cot \theta \sin \psi \cos \phi \partial_{\phi}+\cot \theta \frac{\cos \psi}{\sin \phi} \partial_{\psi}, \\
& X_{2}=\sin \phi \cos \psi \partial_{\theta}+\cot \theta \cos \phi \cos \psi \partial_{\phi}-\cot \theta \frac{\sin \psi}{\sin \phi} \partial_{\psi}, \\
& X_{3}=\cos \phi \partial_{\theta}-\cot \theta \sin \phi \partial_{\phi}, \\
& X_{4}=\sin \psi \partial_{\phi}+\cot \phi \cos \psi \partial_{\psi}, \\
& X_{5}=\cos \psi \partial_{\psi}-\cot \phi \sin \psi \partial_{\psi}, \\
& X_{6}=\partial_{\psi} .
\end{aligned}
$$

The KVs $X_{1-3}$ do not satisfy conditions (24), (25) and hence do not provide Noether symmetries. Therefore the Noether symmetries are the KVs $X_{4-6}$ with corresponding gauge functions $A^{i}(\theta, \phi, \psi)=A^{i}(\phi, \psi)$, where $A_{, i}^{i}(\theta, \phi, \psi)=0$.

We note from Corollary 3 that the minimal surface Lagrangian without the constraint of constant volume (i.e. $\lambda=0$ ) admits all the $\mathrm{KVs} X_{1-6}$ of $S^{3}$ as Noether symmetries. 
M. Tsamparlis, A. Paliathanasis \& A. Qadir

\subsection{Schwarzschild spacetime}

Consider the empty static spherically symmetric spacetime

$$
d s^{2}=-e^{\nu(R)} d t^{2}+d R^{2}+e^{\mu(R)}\left(d \theta^{2}+\sin ^{2} \theta d \phi^{2}\right) .
$$

We consider the surface $R=R(t, \theta, \phi)$ and apply the above analysis. The metric

$$
h_{i j}=\operatorname{diag}\left(-e^{\nu(R)}, e^{\mu(R)}, e^{\mu(R)} \sin ^{2} \theta\right)
$$

gives $|h|=e^{\nu} e^{2 \mu} \sin ^{2} \theta$. Therefore the Lagrangian of the minimal surface enclosing a constant volume in this space is

$$
L=\sqrt{e^{\nu+2 \mu} \sin ^{2} \theta-e^{2 \mu} \sin ^{2} \theta R_{, t}^{2}+e^{\nu+\mu} \sin ^{2} \theta R_{, \theta}^{2}+e^{\nu+\mu} R_{, \phi}^{2}}+\lambda \sin ^{2} \theta \int e^{\nu+2 \mu} d R .
$$

The static spherically symmetric spacetime admits four Killing vectors which are the generators of the $s o(3)$ algebra and the vector $\partial_{t}$. These vectors are independent of the variable $R$. Therefore from Theorem 2 these KVs are the Noether symmetries of this Lagrangian with vanishing gauge fields $A^{i}$.

Our examples are all consistent with the statement given as Theorems $1-3$ in 11] and can, to that extent, be taken as support for those theorems.

\subsection{FRW spacetime}

Consider the surface $t=t(x, y, z)$ in a FRW spacetime

$$
d s^{2}=-d t^{2}+a^{2}(t)\left(d x^{2}+d y^{2}+d z^{2}\right) .
$$

The metric $h_{i j}=\operatorname{diag}\left(a^{2}, a^{2}, a^{2}\right),|h|=a^{6}$. Therefore the minimal surface Lagrangian is

$$
L=\sqrt{a^{6}+a^{4}\left(t_{, x}^{2}+t_{, y}^{2}+t_{, z}^{2}\right)}+\lambda \int a^{3}(t) d t .
$$

For a general function $a(t)$, this spacetime admits the vector fields $\partial_{x}, \partial_{y}, \partial_{z}$, (translations) and the $S O(3)$ (rotations of the Euclidian space in Cartesian coordinates) as KVs. These vector fields are independent of the variable $t$. Therefore these KVs are Noether symmetries of the FRW Lagrangian with vanishing gauge fields $A^{i}$.

In order to show how the Noether point symmetries are applied we select the special case of the dust universe; For this spacetime $a(t)=t^{\frac{2}{3}}$. The minimal surface equation in this spacetime which results from the Lagrangian (48) is a second order PDE which independent variables $\{x, y, z\}$. We select the two Noether symmetries $\partial_{y}, \partial_{z}$. Because these Lie symmetries (Noether point symmetries are also Lie symmetries) commute i.e. $\left[\partial_{y}, \partial_{z}\right]=0$ the reduction of the equation by one of these vectors will lead to an equation which will admit the remaining vector as a Lie symmetry. As it is well known [13/14 this reduction is realized by means of the zero order invariants of the symmetry vector.

The zero order invariants of $\partial_{y}$ which are $\{x, z, r(x, z)\}$ hence reduction by $\partial_{y}$ reduces the equation to the variables $x, z$. Further reduction of the reduced equation 
with the second Lie symmetry $\partial_{z}$ whose zero order invariants are $\{x, s(x)\}$ reduces the ODE to an ODE, which turns out to be

$$
3 s^{\frac{8}{3}} s_{, x x}-8 s^{\frac{5}{3}} s_{, x}^{2}-6 s^{3}-3 \lambda\left(s^{2}+s^{\frac{2}{3}} s_{, x}^{2}\right) \sqrt{s^{4}+s^{\frac{8}{3}} s_{, x}^{2}}=0
$$

where $t(x, y, z)=s(x)$. Similarly one can apply the remaining symmetry vectors in order to reduce the minimal surface equation. We note that the classification of the invariant solutions of the minimal surface equation in the $E^{3}$ can be found in [910].

\section{Conclusion}

We have determined the minimal surface Lagrangian in an $n$-dimensional Riemannian space. Furthermore we have shown that the Noether point symmetries of this Lagrangian are elements of the Killing algebra of the space where Lagrange equations are stated. The determination of Noether symmetries is a useful tool because they provide conservation laws which can be used in order to reduce the order of the differential equation. This implies that if there are enough Noether symmetries it may be possible that one is able to find the solution of the minimal surface equation by means of quadratures. We have demonstrated the results by a number of examples and it has been shown that they agree with those of the literature, whenever applicable. A possible extension of the present study would be the determination of the Lie point symmetries of the minimal surface equation and the use of the first order invariants in the expression of this equation in terms of invariant coordinates.

\section{Acknowledgments}

The authors thank the anonymous referee for the useful remarks and suggestions which significantly improved this work. AP acknowledge financial support of INFN (initiative specifiche QGSKY, QNP, and TEONGRAV).

\section{References}

[1] A. V. Aminova and N. Aminov, "Projective geometry of systems of differential equations: generalconceptions", Tensor 62 (2000) 65.

[2] T. Feroze, F.M. Mahomed and A. Qadir, "The connection between isometries and symmetries of geodesic equations of the underlying spaces", Nonlinear Dynamics $\mathbf{4 5}$ (2006) 65.

[3] M. Tsamparlis and A. Paliathanasis, "Lie symmetries of geodesic equations and projective collineations", Nonlinear Dynamics 62 (2010) 203.

[4] M. Tsamparlis and A. Paliathanasis, "Lie and Noether symmetries of geodesic equations and collineations", Gen. Relativ. Gravit. 42 (2010) 2957; (arXiv:1101.5769).

[5] Y. Bozhkov and I.L Freire, "Special conformal groups of a Riemannian manifold and Lie point symmetries of the nonlinear Poisson equation", J. Differential Equations 249 (2010) 872.

[6] I.L. Freire, "On the paper 'Symmetry analysis of wave equation on sphere' by $\mathrm{H}$. Azad and M.T. Mustafa", J. Math. Anal. Appl. 367 (2010) 716. 
[7] A. Paliathanasis and M. Tsamparlis, "Lie point symmetries of a general class of PDEs: The heat equation", Journal of Geometry and Physics 62 (2012) 2443; (arXiv:1210.2038).

[8] A. Paliathanasis and M. Tsamparlis, "The geometric origin of Lie point symmetries of the Schrodinger and the Klein Gordon equations", Int. J. Geom. Methods Mod. Phys. 11 (2014) 1450037 (arXiv:1312.3942).

[9] N. Bila, Lie Groups Applications to Minimal Surfaces PDE, Proceedings of the Workshop on Global Analysis, Differential Geometry and Lie Algebras, BSG Proceedings" 3 (1999), G. Tsagas (ed.), Geometry Balkan Press, 196.

[10] A. Peterson and S. Taylor, "Locally Isometric Families of Minimal Surfaces, Balkan Journal of Geometry and Its Applications" 13 (2008) 80.

[11] A. Aslam and A. Qadir, "Noether Symmetries of the Area-Minimizing Lagrangian", J. Applied Mathematics 2012 (2012) 532690.

[12] N.H. Ibragimov, Transformation Groups Applied to Mathematical Physics, D. Reidel Publishing Co, Dordrecht (1985).

[13] H. Stephani, "Differential Equations: Their Solutions Using Symmetry", Cambridge University Press, New York, (1989).

[14] G.W. Bluman and S. Kumei, "Symmetries of Differential Equations", SpringerVerlag, New York, (1989) 